Geopolítica(s) Revista de estudios sobre espacio y poder ISSN: 2172-3958

http://dx.doi.org/10.5209/GEOP.57886

\title{
Eppur continua a muoversi. La dinámica colonial del conocimiento científico a través de las revistas y los índices de impacto ${ }^{1}$
}

\author{
Sergio D’Antonio Maceiras²
}

Recibido: 2 de noviembre de 2017 / Aceptado: 22 de abril de 2018

Resumen. Cuando se trata la geopolítica del conocimiento suele hacerse referencia a la ciencia, las universidades y sus modelos de conocimiento, pero en la actualidad las carreras académicas giran en torno a las publicaciones científicas y su impacto. Por ello el objetivo principal de este trabajo es analizar la dinámica y evolución de estos agentes. Para ello se utilizan como marcos de referencia las epistemologías del sur, y dentro de este marco se interrogan y ponen en cuestión las diferentes afirmaciones que la ciencia ha hecho sobre sí misma, y sobre el método de difusión hegemónico en la actualidad: el artículo científico. Inicialmente se describe la evolución de la ciencia y cómo las revistas y los índices han cobrado su protagonismo actual. Posteriormente, se analizan una serie de alternativas actuales, interrogándoles si amplían el horizonte de posibilidades o, por el contrario, ahondan en las dinámicas colonialistas y elitistas. Finalmente se muestra por qué en la actualidad las alternativas se construyen como posibles en tanto que pueden ser integradas por el sistema hegemónico de gestión del conocimiento; mientras que las resistencias ofrecen estrategias y marcos prácticos de acción con capacidad para plantear y ampliar nuevos horizontes de posibilidades.

Palabras clave: revistas científicas; índices de impacto; colonialismo; editoriales comerciales; geopolítica del conocimiento.

\section{[en] Eppur Continua a Muoversi. The Colonial Dynamics of Scientific} Knowledge in Journals and Impact Factors

\begin{abstract}
Discussions about the geopolitics of scientific knowledge usually make reference to science, universities and their learning models, yet today academic careers are rather centred on scientific publications and their impact. Hence the main purpose in this work is to analyse this change of paradigm. The articles places meditations on Southern epistemologies as a framework of reference from which to critically revise how science has traditionally defined itself and the hegemonic method for knowledge dissemination: scientific articles. To begin with, the article describes the evolution of science and how journals and impact factors have gained their current status. Next, the article ponders over a number of alternatives to the current model. It addresses the question of whether such alternatives widen the range of possibilities, or else deepen extant colonialist and elitist dynamics. Lastly, it shows why today the exploration of alternatives is linked to their potential for integration in the hegemonic system of knowledge management, while resistances offer strategies and practical frameworks for action that open up new horizons.
\end{abstract}

1 Agradezco a quienes evaluaron, corrigieron y editaron versiones previas de este texto. Sus sugerencias han contribuido notablemente a su mejora.

2 Facultad de Trabajo Social, Universidad Complutense de Madrid

E-mail: sergiodantonio@ucm.es 
Keywords: scientific journals; impact factor; colonialism; publishing houses; the geopolitics of knowledge.

\section{[pt] Eppur continua a muoversi. A dinâmica colonial do conhecimento científico através das revistas e os índices de impacto}

Resumo. Quando se trata a geopolítica do conhecimento costuma se fazer referência à ciência, as universidades e seus modelos de conhecimento, mas na atualidade as carreiras académicas giram em torno das publicações científicas e seu impacto. Por isso o objetivo principal deste trabalho é analisar a dinâmica e evolução destes agentes. Para isso se utilizam como marcos de referência as epistemologias do Sul, e dentro deste marco se interrogam e põem em questão as diferentes afirmações que a ciência tem feito sobre si mesma, e sobre o método de difusão hegemónico na atualidade: o artigo científico. Inicialmente descreve-se a evolução da ciência e como as revistas e os índices têm cobrado seu protagonismo atual. Posteriormente, analisam-se uma série de alternativas atuais, interrogandolhes se ampliam o horizonte de possibilidades ou, pelo contrário, afundam nas dinâmicas colonialistas e elitistas. Finalmente mostra-se porquê na atualidade as alternativas constroem-se como possíveis enquanto podem ser integradas pelo sistema hegemónico de gestão do conhecimento; enquanto isso as resistências oferecem estratégias e marcos práticos de ação com capacidade para propor e ampliar novos horizontes de possibilidades.

Palavras-chave: revistas científicas; índices de impacto; colonialismo; editoriais comerciais; geopolítica do conhecimento.

Sumario. Introducción. 1. El avance de la ciencia gerencial y técnica en el siglo XX. 2. La aparición de las revistas científicas, editoriales comerciales y los índices de impacto 3. La crisis permanente: alternativas y resistencias en el siglo XXI. 3.1. Las alternativas "integradas": acceso abierto, pago por publicación y métricas alternativas. 3.2. Las "apocalípticas": resistencias, ampliación del espacio e ironías. 4. Contra las alternativas. Conclusiones. Eppur continua a muoversi. Bibliografía.

Cómo citar: D’Antonio Maceiras, Sergio (2018) “Eppur continua a muoversi. La dinámica colonial del conocimiento científico a través de las revistas y los índices de impacto". Geopolítica(s). Revista de estudios sobre espacio y poder, vol. 9, núm. 1, 113-136.

\section{Introducción}

Cuando se trata la geopolítica del conocimiento es ineludible referirse a la ciencia, las universidades y sus modelos de conocimiento. Pero, ¿hay alguna aproximación práctica que, deudora de las epistemologías del sur (Santos \& Meneses, 2014), pueda aportar luz sobre la reproducción del conocimiento en las capas más materiales de su producción? En este trabajo se propone que el análisis de las revistas científicas muestra las dinámicas de colonización ${ }^{3}$ del conocimiento, y ayuda a comprender por qué las alternativas propuestas profundizan los discursos coloniales en el Norte y Sur globales.

3 A lo largo de este trabajo se utilizará el término colonial para hacer referencia a la situación de “dependencia histórico-estructural entre la metrópoli y las colonias, entre el imperialismo y el colonialismo en todos los planos: económico, político, epistémico y en la formación de subjetividades" (Mignolo, 2005: 49). En otro punto de su obra (Mignolo, 2013) el mismo autor maneja el mismo concepto abordándolo desde una perspectiva de la resistencia que aquí no se desarrolla. 
Siguiendo a Santos (Santos \& Meneses, 2014), el pensamiento occidental moderno se caracteriza por una serie de divisiones, líneas "abismales", que delimitan aquello que existe de aquello que desaparece de la realidad y se convierte en no existente. El imperialismo se ha construido apoyándose en la universalización del sujeto de conocimiento occidental europeo (Castro-Gómez, 2004; 2010), relegando a la no existencia al resto de saberes hasta llegar al momento actual en el que la ciencia es la única forma válida para explicar, predecir y comprender la realidad (Santos, 2010b). Partiendo de unas líneas demarcadoras que establecen si un conocimiento es científico - este lado de la línea — o no —el otro lado de la línea- el conocimiento científico progresivamente ha ido marginando otros saberes al tiempo que ha construido una jerarquía dentro de este tipo de saber. A este lado de la línea - es decir, dentro del conocimiento científico - la ciencia progresa gracias a una orientación claramente metrificadora y deudora del "dato". Apoyada en el modelo de difusión científica actual, no sólo perpetúa delimitaciones abismales geográficas y coloniales, sino que también impulsa otra serie de líneas abismales invisibles que tienen a producir un tipo específico de conocimiento como "el bueno".

Por estas razones, para escribir sobre revistas científicas y su papel en la geopolítica del conocimiento es necesario establecer los límites dentro de los cuales se moverá la interpretación y las estrategias discursivas que se emplearán. Este trabajo comienza recorriendo la evolución de la ciencia a lo largo del siglo XX para mostrar cómo revistas e indicadores de impacto alcanzan su protagonismo actual. Posteriormente se analizan las diferentes alternativas y resistencias, con el fin de visibilizar las demarcaciones que el conocimiento científico establece. Finalmente, utilizando el marco de referencia de las teorías descoloniales y el solucionismo, se persigue interrogar las distintas dinámicas sobre el cumplimiento de sus promesas, analizando sus desarrollos concretos, las líneas que demarcan y las consecuencias que generan (Santos, 2010a; 2010b). A través de este recorrido se llega a una explicación de las dinámicas que retroalimentan un tipo de conocimiento científico cada vez más colonial y específico del Norte Global.

\section{El avance de la ciencia gerencial y técnica en el siglo $X X$}

Cuando se mencionan los valores intrínsecos a los que debería aspirar la ciencia la teoría de los CUDEOS de Merton \& Storer (1985) continúa siendo el referente más aceptado. Se trata de cuatro valores normativos básicos: el comunismo, que establece la propiedad común (pública) de los conocimientos científicos a cambio del prestigio; el universalismo, por el cual las afirmaciones científicas serán evaluadas impersonalmente y no en función de la raza, clase, género, religión, nacionalidad, etc.; el desinterés generalizado, que conduce a que el trabajo científico sea recompensado - es decir, validado - en función únicamente del prestigio; y finalmente el escepticismo organizado, según el cual todas las ideas deben ser probadas y estar sujetas a rigurosos escrutinios por parte de la comunidad científica. Estos valores han sido ampliamente contrastados y no puede afirmarse que reflejen la práctica científica cotidiana, respecto a las revistas científicas estos valores son refutables sencillamente. El comunismo es poco menos que papel mojado; el conocimiento científico actualmente se encuentra en manos de grandes conglomerados editoriales 
que cobran cantidades importantes de dinero para su acceso y los artículos de acceso abierto, a los que se puede acceder gratuitamente, provienen cada vez más de revistas que cobran a los autores por publicar. El universalismo queda cuestionado con el anglo-europeísmo del conjunto de revistas top, principalmente publicadas en inglés y orientadas casi exclusivamente a los intereses de investigación del Norte Global. Respecto al desinterés generalizado, más allá de que el sistema de publicaciones actual puede considerarse como un gran mercado neoliberal, sus validaciones - el prestigio académico — son progresivamente menos relevantes que las ofrecidas por su contrapartida privada: el mercado. En la actualidad trabajar en la Universidad no otorga más prestigio que hacerlo en Facebook, Google o Tesla y tampoco un salario equiparable ${ }^{4}$. Finalmente, respecto del escepticismo organiza$d o$, esbozar todas las ocasiones en las que no se cumple sería interminable. Desde las ciencias "puras" - las buenas - siempre se menciona burlonamente el caso Sokal $^{5}$, si bien dentro de las ciencias "puras" cada año aumentan los artículos que se retiran de circulación por distintos tipos de errores o malas prácticas ${ }^{6}$.

Desde sus inicios la ciencia ha estado íntimamente relacionada con las diferentes formas de gobierno y poder que se fueron sucediendo en Europa. Entre los siglos XVII y XIX ya claramente era una herramienta al servicio de los gobiernos respecto a la planificación y gestión de las ciudades, las medidas de higienización y de control de la población (Foucault, 2014). En el siglo XIX el Imperialismo impuso estos ideales en las colonias (Castro-Gómez, 2010). Este recorrido tiene corolario en la importancia de la técnica en los inicios del siglo XX y su protagonismo en las dos Guerras Mundiales. Hasta este momento, puede considerarse que la ciencia había sido una herramienta en manos de un "ideal superior" a lo largo de toda la historia. El Antiguo Régimen, el Imperialismo, el nazismo y las democracias en su faceta imperialista primero, y de lucha contra el fascismo después, utilizaron el potencial y utilidad de la ciencia para sus fines. Con el final de la Segunda Guerra Mundial y la constitución de los dos bloques la posición de la ciencia cambia. En 1945 Vannevar Bush publica un ensayo (1945a) y un informe para el Presidente de los Estados Unidos (1945b) donde se sentarán las bases de la sociedad del conocimiento y algunas ideas clave para el futuro: el informe sostiene que el desarrollo técnico ha sido en gran medida lo que ha permitido la victoria aliada, y la sociedad que lo domine obtendrá superioridad respecto a las otras. Por ello, es imprescindible que el Gobierno invierta en conocimiento e investigación (civil y militar) también en tiempos de paz. Para ello es fundamental la inversión pública en una investigación básica que, "sin tener la mirada en fines prácticos", sea regulada por una agencia independiente que rinda cuentas al gobierno y asegure los controles democráticos. Así, en 1945 se sientan las bases de una nueva sociedad en la cual el co-

4 Por ejemplo, el trabajador medio en Google España gana $€ 145.000$ al año, una cifra impensable para cualquier docente y científico público en España. Ver URL:

$<$ https://elpais.com/economia/2017/09/26/actualidad/1506450261_564988.html>. (Consultado el 12-1-18).

5 En justicia, el asunto es ciertamente tragicómico. El escándalo se produjo cuando un físico envió a una prestigiosa revista de Ciencias Sociales, centrada en los debates posmodernos y la Guerra de las Ciencias, un artículo completamente inventado, sin sentido y con un lenguaje abstruso que acabó publicado (Sokal, 1996; Sokal et al., 1998).

6 Para un análisis actual de las razones ver (Fanelli, 2009; Fanelli, 2014; Fanelli et al., 2015). También hay una página web que actualiza permanentemente este tema: <http://retractionwatch.com/>. (Consultado el 25-517). 
nocimiento tiene que ser eficiente e innovador (Echeverría, 2014), estar fuertemente financiado por el Gobierno y, por tanto, debe rendir cuentas a la sociedad. Evidentemente en todo este proceso se contará con la iniciativa privada. El bloque occidental promovía una sociedad libre, democrática y capitalista en la que la corporación privada, con el Estado como agente principal, tenía un papel fundamental en la sociedad.

Así, la ciencia gestionada gerencialmente por mandos intermedios, agencias, comisiones y personal técnico (Burnham, 1945; Orwell, 1955) será el único tipo de conocimiento válido, sin un referente superior como anteriormente. Para ambos bloques, tanto el occidental como el soviético, el progreso a través del conocimiento científico-técnico-gerencial será la única vía para mejorar la sociedad. ¿En qué consistía ese camino? Sólo la ciencia administrada técnica y gerencialmente podría decidirlo. En la práctica este modelo impulsaba aún más el conocimiento técnico metrificable, institucionalizaba la aplicabilidad y una doble vía para validar el conocimiento. Las corporaciones privadas, centradas en la investigación aplicada para la gran industria militar, validaban sus productos tanto en el ámbito militar como en sus aplicaciones en el mercado civil de consumo, es decir dinero. Pero al no tener una aplicabilidad clara e inmediata la investigación básica no podía validarse directamente en el mercado. Históricamente la forma de reconocer el valor del trabajo había sido el prestigio - una validación tan efectiva como profundamente patriarcal y colonial - expresado a través del reconocimiento por parte de la comunidad científica.

Pero el dinero y el prestigio por sí mismos no podían soportar todo el andamiaje necesario para sostener este modelo. Dentro del ámbito privado el conocimiento y las innovaciones militares permanecieron secretas, y aquellas que podían ser rentabilizadas en el mercado civil fueron protegidas por leyes de propiedad industrial, intelectual y patentes cada vez más restrictivas. Esto fomentó la creación de un mercado en el que se intercambia un tipo concreto de producto: el conocimiento aplicado, de forma que la validación se realiza tanto en función de su utilidad militar como en su aplicabilidad para la sociedad de consumo. Así, la iniciativa privada se aseguraba el posible retorno de sus inversiones no sólo a través de la aplicabilidad de sus productos, sino de un sistema de propiedad intelectual que privatizaba el conocimiento producido con financiación pública. Por otra parte, la validación del conocimiento básico producido por las universidades planteaba un interrogante: ¿cómo medir o evaluar el prestigio? Aquí es donde las revistas científicas, editoriales comerciales y los índices de impacto entrarán en juego. En efecto, con el cambio de modelo dos agentes relativamente secundarios hasta entonces (las revistas científicas y las editoriales comerciales) y uno prácticamente desconocido e incipiente (la cienciometría) cobrarán un protagonismo creciente

\section{La aparición de las revistas científicas, editoriales comerciales y los índices de impacto}

Las primeras dos revistas, la Journal des Sçavans en Francia y las Philosophical Transactions of the Royal Society en Inglaterra, fueron publicadas en 1665 por sendas sociedades científicas. Por aquel entonces la asistencia física a las reuniones era complicada y la correspondencia epistolar se había convertido en una carga de 
trabajo diaria excesiva y lenta (Price, 1986). Las revistas permitían una difusión mayor, de una forma más estructurada y regular (Zuckerman \& Merton, 1971). A la sombra del libro o las monografías, este formato fue ganando popularidad a medida que las sociedades científicas creadas a lo largo del siglo XIX promovían sus propias editoriales y revistas como signo de prestigio. Hacia finales del siglo, si bien constantemente acusadas de paternalismo, con una calidad del papel e impresión habitualmente pésimas y poco respeto a la periodicidad entre otras cuestiones, eran el medio más rápido y eficaz para difundir el conocimiento científico y sus controversias (Larivière et al., 2006). Si bien las editoriales comerciales son más antiguas que las revistas, en concreto éstas nunca habían sido un nicho de negocio importante y hasta mediados del XX cubrían principalmente los huecos dejados por las sociedades científicas. A pesar de ser consideradas menos paternalistas, de una calidad mayor, más rápidas y eficientes, más bien eran las outsiders del sistema (Larivière et al., 2015) y a finales de los años 1950 la mayoría continuaban siendo de sociedades científicas y editoriales universitarias.

Con el cambio de modelo se produjeron transformaciones drásticas; se impuso una comunicación mucho más veloz, eficiente y estructurada, en un contexto cada vez más competitivo para demostrar resultados de investigación y, así, continuar obteniendo financiación. Las revistas resultaron idóneas porque son un formato más adecuado a la gestión gerencial: la periodicidad de una revista es constante, la cantidad de artículos por número también y escribir un artículo es una tarea más "asequible" que un libro. Además el artículo es el formato más utilizado por el tipo de conocimiento que se fomentaba: las ciencias experimentales y técnicas - las buenas - Las agencias gubernamentales de Estados Unidos y el norte de Europa - el primer mundo del primer mundo - comenzaron a favorecer a las editoriales comerciales: resultaban más eficientes, independientes y en consonancia con la relación entre gobiernos, ciencia y capital privado que el modelo proponía. Por su parte, las editoriales comerciales constataron rápidamente que las revistas proporcionaban una fuente de ingresos más regular y abundante que los libros, además de implicar a un público esclavo como se verá más adelante. Así, a partir de los años 1950 comenzó un proceso de expansión basado en la creación exponencial de nuevas revistas para diversificar las fuentes de ingreso. Las universidades, al tener que cumplir una serie de criterios "nuevos" (respetar la periodicidad, aumentar la velocidad de publicación y difusión, establecer sistemas de evaluación por pares, etc.) siguiendo el signo de los tiempos externalizaron sus revistas rentables y abandonaron las que no lo eran (Buranyi, 2017). Así, en consonancia con el signo de unos tiempos en los que el Estado continuaba siendo el agente principal y la iniciativa privada cobraba cada vez más relevancia, comenzó el desplazamiento del espacio de poder por antonomasia del conocimiento: las editoriales comerciales, incentivadas por una nueva situación que las posicionaba con ventaja competitiva y apoyadas en las leyes de propiedad intelectual, progresivamente se fueron convirtiendo en las propietarias del conocimiento y los porteros (gatekeepers) que controlaban quién y qué se publicaba (Crane, 1967).

Pero todavía no se ha dado respuesta a la pregunta sobre cómo se mide el prestigio científico. La respuesta está en los índices de impacto. En 1961, quince años después del informe de Bush, Eugene Garfield (1955) publica la primera edición del SCI (Science Citation Index). Las suscripciones ya habían comenzado a aumen- 
tar significativamente sus precios, y el índice se ofrecía como un servicio para que el personal bibliotecario conociera las revistas más influyentes de cada disciplina y tomara mejores elecciones de suscripción. Para su elaboración Garfield siguió la estela de una serie de trabajos previos centrados en el análisis cuantitativo de la ciencia (Price, 1951; 1961; 1986) y en la ley formulada por Bradford en $1934^{7}$ (1976). Brevemente, esta ley sostiene que la mayoría de las referencias de una determinada disciplina se concentran dentro de un núcleo reducido de revistas y los círculos alrededor del núcleo reciben una atención exponencialmente inferior. En 1965 Price (1986) había demostrado que en cualquier año y por diversas razones el $35 \%$ de todos los artículos no son citados y el $49 \%$ lo son sólo una vez. Por tanto, analizando el número de citas que recibía una revista se podía calcular su influencia dentro de la disciplina. Cuando en 1975 se publica la primera versión del factor de impacto dentro del SCI ya no se trata de herramientas que ayuden al personal bibliotecario sino de un instrumento capaz de ofrecer una validación del prestigio. Instrumento con el que las agencias de evaluación podían tomar decisiones relevantes respecto a la política científica. Cumplía los criterios propuestos por Bush y respetaba los valores normativos de la ciencia: se trataba de un indicador sintético impersonal, racional y cuantitativo; se elaboraba por un instituto de investigación externo e independiente, el ISI (Institute for Scientific Information); y finalmente, en su formulación se tenían en cuenta los valores mertonianos a los que debía aspirar la comunidad, porque las referencias reflejan la deuda moral e intelectual de un autor respecto a la obra referenciada (Merton \& Storer, 1985; Cole, 2004) ${ }^{8}$; así se asumía que las revistas más citadas serían mejores y al publicar en ellas se habría hecho una mayor aportación al conocimiento científico.

De esta forma la cienciometría, un enfoque secundario de una disciplina marginal hasta los 1930 - la historia y sociología de la ciencia - comienza a convertirse propiamente en una ciencia centrada en la bibliometría; rápidamente institucionalizada con su sociedad científica, el ISI; su propia revista, Scientometrics, fundada en 1978; y con un objeto de estudio claramente definido: la creación de una "ciencia social relativamente dura" que, a través de la metrificación, pueda orientar la política científica. Ciencia que en gran medida ha devorado su objeto de estudio y la disciplina a la que pertenecía. No se trata de una interpretación sesgada o crítica de la historia. Cuatro años después de la primera publicación del JIF, Garfield recapitulará:

En términos coloquiales Morton Malin [miembro del ISI] explica que "parte de la cienciometría consiste en aplicar las técnicas de procesar números (number crunching techniques) al estudio de la ciencia de la ciencia" esto es, a la política científica [...] La investigación cienciométrica tiene amplias aplicaciones. Gobiernos e instituciones de investigación han expresado su interés en aplicar "el

7 Para una explicación un tanto más desarrollada ver URL: $<$ https://es.wikipedia.org/wiki/Ley_de_Bradford $>$. (Consultado el 5-6-17).

8 Se referencia la versión castellana, como la temporalidad es importante en este caso, el libro fue publicado en 1973 en su edición inglesa.

9 Esta frase corresponde a Derek de Solla Price, padre de la cienciometría moderna, en el editorial del primer número de la revista Scientometrics, toda la declaración de intenciones (Price, 1978; Wouters \& Leydesdorff, 1994). 
conocimiento cuantitativo del crecimiento del conocimiento a la gestión de la ciencia". Los indicadores científicos están siendo utilizados en un esfuerzo por comprender la "salud" de la ciencia en varios países. En última instancia, la cienciometría puede emplearse para ayudar a las naciones a tomar decisiones sobre qué áreas de conocimiento necesitan financiación (1979: 313-314).

En consecuencia, si a través de las editoriales comerciales se externaliza el conocimiento científico, con el índice de impacto se externaliza, a través del ISI, la validación de los resultados de este conocimiento. Ambos desarrollarán el mismo modelo de negocio. Mientras que las editoriales comerciales rápidamente comenzaron a aumentar la oferta de servicios a través de revistas cada vez más especializadas para aumentar los costes de suscripción, el ISI diversificó sus productos incluyendo índices disciplinares, regionales y de segunda categoría ${ }^{10}$. Esta de externalización de servicios por un lado, y aumento por la vía de la diversificación por el otro, se agudizará a partir de finales de los años 1970, cuando este proceso conduzca a que el Estado deje de ser el agente principal en el establecimiento de reglas dentro del escenario político-económico para ser uno más. Por ello no es extraño que en 1984 el conglomerado de medios Thompson-Reuters compre el ISI y, en 2017, ésta cree una nueva spin-off (Clarivate Analytics) que se ocupe del negocio de analíticas y gestión sobre ciencia y patentes.

Dejando de lado el eje temporal, el colonialismo del conocimiento científico se basa en la invisibilización de sus contextos de creación a través de criterios y técnicas de justificación que naturalizan las desigualdades materiales (Castro-Gómez, 2010). Creado por las sociedades del Norte Global, este conocimiento avanzará marginando al resto de saberes (Santos, 2010a). En tanto se trataría de un conocimiento objetivo, imparcial e igualitario, se ocultarán las condiciones materiales de producción, así como sus posibilidades de reproducción. Con la institucionalización de métricas universales a través de una serie de servicios, técnicas, estadísticos y procesos de gestión, estas desigualdades estructurales se ocultan, naturalizan y justifican al mismo tiempo que se materializan en tres líneas abismales. La primera invisibilizará, eliminándolos de la esfera pública, todos los conocimientos que no sean científicos ni se hayan validado en publicaciones científicas. De este lado de la línea, es decir dentro del conocimiento científico, la segunda línea introducirá un abismo geográfico porque, como era previsible, los núcleos de las disciplinas estaban ocupados por revistas del primer mundo del Norte Global (EE UU, Inglaterra, Alemania, Países Bajos y Francia) junto a un segundo círculo de países periféricos del Norte Global (España, Italia, China, Rusia y Japón) y una clara infrarrepresentación y sesgo de las revistas de países en desarrollo que ya fue criticada antes del fin de siglo (Garfield, 1997). Finalmente, una tercera línea cognitiva que terminará de dar forma a una ciencia con características muy concretas: en tanto las editoriales comerciales centraron su expansión en las disciplinas técnicas y computacionales (Buranyi, 2017), el estilo de redacción pasó de un formato más narrativo a una presentación secuencial, lógica e higiénica de los pasos seguidos para alcanzar una conclusión (Gross et al., 2002). Esto fomentó una "cientificización" de las ciencias sociales, las humanidades, la filosofía y el derecho, disciplinas que tenían en el

10 Repiso \& Torres-Salinas (2016) resumen esta situación de inflación de índices y productos diferenciados. 
libro su formato por excelencia y cuyo estilo era más narrativo y no lógicoprocesual-higiénico. Al igual que con los países periféricos, ésta infrarrepresentación de las ciencias sociales y humanidades no ha dejado de incrementarse.

La supuesta necesidad de un validador único para toda la ciencia global, producido por dos corporaciones del Norte Global, resulta en una imposición "sutil" que discrimina a los países del Sur Global al mismo tiempo que continúa ocultando la colonialidad del proceso de creación, elaboración y medición del conocimiento. Las consecuencias son la sobre-representación del inglés dentro de la literatura científica y el foco en los temas de investigación que interesan al Norte Global (el cáncer, la biomedicina o la robótica) cada vez más alejados de problemas locales o invisibilizadas por ser propias del Sur Global (la malaria, el ébola y el SIDA son las más sangrantes) pero también análisis económicos, sociales, etc. En definitiva, en la medida que el prestigio se valida a través de índices internacionales, y la mayoría de revistas indexadas son publicadas en el Norte Global y en inglés, sistemáticamente se discriminan o minusvaloran las líneas de investigación sobre temas locales o problemas que afectan a la inmensa mayoría de población mundial que vive en el Sur Global. Así las revistas científicas y los índices de impacto son un pilar fundamental a partir del cual pivotan, dentro de la ciencia misma, un conjunto de prácticas que determinan y orientan los temas, los tipos de investigación y quiénes pueden acceder a ella, qué tipo de profesorado universitario llega a impartir clases, qué se enseña y qué tipo de ciencia se lleva a cabo ${ }^{11}$. Además, si la ciencia es el único tipo de conocimiento válido en la actualidad, la otra cara de la moneda es que se trata de un conocimiento profundamente endogámico y, en la permanente persecución de la excelencia, cada vez más pequeño y cerrado sobre sí mismo. Es decir, cada vez más endogámico. Por ello la humildad de este objeto de estudio es falsa y cínica. Si no se matan ideas, a través de este círculo vicioso "impersonal" sí se consiguen callar, lo que hoy en día es parecido.

\section{La crisis permanente: alternativas y resistencias en el siglo XXI}

A lo largo de esta historia han surgido numerosas críticas a este sistema. Y si bien se han desarrollado resistencias respecto a la hegemonía de las grandes editoriales comerciales, las alternativas al SCI y SCOPUS han continuado siendo vicarias de estos. En consecuencia, es pertinente aproximarse a las dinámicas oligopólicas de las grandes editoriales y los índices de impacto para, posteriormente, analizar las distintas alternativas siguiendo la distinción de Umberto Eco entre las "integradas" y las "apocalípticas" (2015). Aunque esta distinción no respete precisamente las caracterizaciones realizadas por el gran semiólogo italiano, sí mantiene cierto espíritu de semejanza. Grosso modo, las alternativas son "integradas" en tanto han sido incorporadas al modelo hegemónico de revistas científicas, e involucran un cierto optimismo respecto a la capacidad del sistema para ser mejorado sin que se alteren sus presupuestos de funcionamiento ni sus relaciones de producción. Por el contra-

11 En Estados Unidos el proceso por el cual las élites han ocupado la Universidad a partir de los años 1970 ha sido descrito por Naomi Klein (2010). 
rio, las resistencias "apocalípticas" no han sido integradas por el sistema y son vistas como amenazas por algunos agentes del modelo. En este sentido, se trata de un criterio dinámico en función de su incorporación dentro del discurso de los agentes hegemónicos dentro del modelo de difusión científica.

La evolución del sistema de publicaciones condujo a un proceso de concentración oligopólico en el que cinco editoriales comerciales (RELX, Taylor \& Francis, Wiley-Blackwell, Springer y Sage, las big-five) controlaron progresivamente el mercado de revistas. Sólo ellas publicaron algo más del 20\% de los artículos recogidos en la Web of Science (WoS) —es decir, los buenos - en 1973 y el 30\% en 1996, alcanzando el 70\% en ramas como las Ciencias Sociales (Larivière et al., 2015). Con la masificación de Internet a lo largo de la última década del siglo pasado se pensaba que las cosas cambiarían. La red podía aportar una estructura técnica descentralizada y una arquitectura legal abierta gracias a las comunidades de software libre. Quizás por ello la revista Forbes se preguntaba en 1995 si RELX (Elsevier), después de sus casi 400 años de historia, no sería la primera víctima de Internet (Hayes, 1995). En 1999, la Declaración de Budapest reclamó el acceso gratuito y público de todas las investigaciones afirmando "que en el siglo XXI la ciencia debe convertirse en un bien compartido solidariamente en beneficio de todos los pueblos" (1999); con este objetivo nacen en 2001 las licencias Creative Commons, dos años después aproximadamente un millón de materiales tenían una de estas licencias ${ }^{12}$. Pero las big-five aumentaron su cuota: tres años después, en 2006, la mitad de los artículos publicados en la WoS eran de sus revistas y en 2013 subió al 53\%. En 2015 en un artículo titulado "Elsevier lidera el negocio que Internet no pudo matar" el Financial Times recomendaba invertir en RELX (Cookson, 2015). ¿Qué sucedió?

Brevemente, los Estados habían perdido la capacidad de ejercer control o influencia sobre este sistema e Internet, esa red "de pasado militar, presente anarquista y futuro comercial" (Gray, 2002), que "con su estructura abierta lo solucionaría todo", no alteró las raíces del sistema: las revistas y los artículos científicos, es decir la propiedad de los medios de producción. Por otra parte, Internet sí permitió una reducción muy significativa de los costes y tiempos editoriales fundamentalmente por la progresiva eliminación del papel. Gracias a ello comenzó el último incremento exponencial del número de revistas; principalmente editadas por universidades y siguiendo la Declaración de Budapest, la mayoría se publican con licencias de acceso abierto. Cuestión que beneficia a las big-five porque les permite incrementar el valor económico y simbólico de sus catálogos.

\subsection{Las alternativas "integradas": acceso abierto, pago por publicación y mé- tricas alternativas}

El giro hacia la publicación digital benefició a las big-five entre otras cosas porque les permitió modificar el sistema de suscripciones. Con el papel, la suscripción

12 Ver URL: <https://creativecommons.org/about/history/>. (Consultado el 10-05-17). 
implicaba recibir el ejemplar físico de la revista y si al año siguiente no se continuaba la biblioteca conservaba la revista. Con la masificación de Internet se pasó a un modelo de pago por acceso — como Netflix o Spotify — en el que se paga un canon por acceder a los contenidos y si se deja de pagar se pierde todo, no hay nada en los estantes. En consecuencia, el acceso abierto podría ser una solución respecto a las publicaciones presentes y futuras, pero mientras se continúen aplicando las leyes actuales de propiedad intelectual nunca resolverá el problema de las suscripciones a las revistas ya editadas. Por otra parte, a lo largo de los años las big-five crearon o compraron las mejores revistas y las más rentables, que en su mayoría continúan siendo cerradas, con acceso mediante suscripción. Respecto a las revistas nuevas, estas tienen que atravesar un proceso ${ }^{13}$ tedioso e incierto de años para poder ser parte de alguno de éstos índices, mientras que las publicadas por las big-five habitualmente entran mucho más rápido o desde su misma creación. Por esta razón, las Universidades que pueden permitírselo pagan o coeditan con las big-five para obtener desde el primer momento revistas indexadas. Se trata de una dinámica viciosa en donde la calidad en gran medida está determinada por las mismas partes involucradas, generando un círculo vicioso.

El modelo de las revistas nuevas, abiertas y editadas por universidades implica renunciar a los ingresos por suscripciones, históricamente su fuente principal, y mantener sus publicaciones apoyándose en el trabajo voluntario y ocasionalmente en financiación institucional. La crisis económica que afectó a Europa y Estados Unidos a partir de 2008 recortó drásticamente esta financiación y muchas revistas buscaron nuevas vías. En este contexto se empieza a popularizar el modelo de Pago por Publicar (PPP, o APC, por sus siglas en inglés). Originalmente este modelo perseguía cubrir los costes operativos de los artículos invirtiendo la carga y cobrando por publicar. Los análisis realizados estiman que si bien el coste de un artículo es de aproximadamente U\$D100 los precios medios por publicar superaban, en 2014, los U\$D1.000 - diez veces más - y la tendencia era de franco aumento (Solomon \& Björk, 2012; Björk \& Solomon, 2015; Solomon \& Björk, 2016). El resultado es que los costes totales por publicación - lo que las instituciones públicas pagan globalmente - continúa aumentando en lugar de reducirse (Pinfield et al., 2016).

Como ya sucediera con el software libre, el acceso abierto ha conseguido ser progresivamente cooptado en la medida que se han ido desbrozando sus categorías éticas y marcos de referencia más amplios que ponían en cuestión el modelo de las editoriales comerciales. El resultado es un desplazamiento que poco a poco transforma - reaxiomatiza, dirían Deleuze \& Guattari (1985) - el concepto para integrarlo en la dinámica del negocio. Si hace unos años se criticaba a las big-five que

13 Quedan fuera de consideración algunas cuestiones sobre las características del índice de impacto como su arbitrariedad inherente o que sea irreproducible (The PLoS Medicine Editors, 2006). Tampoco es objeto de este trabajo posicionarse sobre el carácter altamente rentable y con poco valor añadido de las editoriales comerciales, cuestión que escandaliza tanto a parte de la comunidad científica como al Deutsche Bank, que lo describió como un "sistema bizarro" de "triple expropiación" (Deutsche Bank, 2005; Beverungen et al., 2012) para los sistemas científicos del Norte Global, de donde provienen estas empresas. 
fueran el principal impedimento para una ciencia libre y no tuvieran revistas en acceso abierto, en la actualidad cada vez publican más en abierto a través de PPP. Ahora la comunidad científica tiene lo que llevaba años reclamando, acceso libre e irrestricto, pero la publicación en revistas "buenas" y de acceso abierto implica con mayor frecuencia pagar unas cantidades cada vez más prohibitivas para el conjunto de la comunidad científica.

Estas dinámicas no serían posibles sin el círculo vicioso entre las big-five y los índices de impacto. Si bien a lo largo de su breve historia la bibliometría ha elaborado una gran cantidad de indicadores para medir la ciencia (Waltman, 2015) hasta ahora se ha puesto el foco en el JIF que continúa siendo el índice de referencia. No obstante en la actualidad existen dos servicios que se han popularizado y ofrecen enfoques diferentes: Google Scholar (GS) y las métricas alternativas (altmetrics por su nombre en inglés). Respecto del primero, Scholar no mide la calidad de las revistas como el JIF sino la cantidad de citas que recibe un artículo a través del índice-h. Al rastrear toda la red, además de las revistas de alto impacto, también recoge las citas de revistas "periféricas", tesis doctorales, repositorios y libros. Se trata de un enfoque mucho más abierto e inclusivo. Sin embargo, esta herramienta plantea algunos inconvenientes. Los estudios muestran que si bien es capaz de capturar un número mayor de citas lo hace a costa de la precisión: duplica resultados, atribuye autorías erróneamente y la extracción masiva de información para su depuración y análisis es más compleja (Mingers \& Leydesdorff, 2015; Harzing \& Alakangas, 2016). Además, más que un servicio se trata de una característica añadida al servicio principal de búsqueda bibliográfica utilizando los algoritmos secretos de Google, lo que no supone un avance en transparencia. Por otra parte las métricas alternativas (altmetrics) en los últimos años están cobrando cada vez más importancia. Surgidas en 2010 además de las citas también incluyen las menciones en distintos medios sociales como revistas especializadas, blogs científicos, Wikipedia, Twitter, Facebook; los "favoritos", recomendaciones de sitios y los artículos incluidos en gestores de referencias. Si bien se trata de un servicio cada vez más presente en la bibliografía y las revistas, sus creadores insisten en que su objetivo de complementar, no reemplazar, las métricas oficiales.

Tanto GS y las altmerics amplían las posibilidades de evaluación y descentralizan la recogida de datos, pero no resuelven la mayoría de los problemas al tiempo que generan otros: GS, al igual que el JIF, continúa siendo citacentrista porque se basa en el número de citas recibidas; uno con una muestra artificialmente elitista y precisa, otro con una muestra tanto más amplia como imprecisa. Por su parte las altmetrics, ampliando la cantidad de fuentes, socializan el citacentrismo dentro del concepto más amplio de menciocentrismo, haciendo que el impacto pivote alrededor de las menciones que se recibe en los medios sociales. Ambos mantienen el problema de la orientación de las citas, algo que rápidamente se demostró que no era así (MacRoberts \& MacRoberts, 1996; MacRoberts, 1997) y que se amplifica con las altmetrics, porque en medios sociales las menciones en tono burlesco, satírico o como crítica furibunda alcanzan el 33\% (Sugimoto, 2015). En definitiva, no se trata sólo de pagar por publicar, sino después hacerse auto-publicidad y promo- 
ción a través de todos los medios sociales posibles. No importa si los resultados generan buenas o malas críticas porque ningún indicador lo distingue. Más aún, sabiendo que en redes sociales se difunde mucho más eficientemente la burla y la crítica que el reconocimiento: "Que hablen de mí, aunque sea mal".

A través de la igualdad de oportunidades estas alternativas eliminan la diversidad de la realidad, y en su evolución terminan profundizando las brechas entre el Sur y Norte globales. Los PPP ahondarán la enorme desigualdad que existe no sólo entre el Norte y Sur globales, sino también dentro de los temas de investigación y desarrollo locales del Sur Global simplemente porque no son temas que interesen al Norte Global. La socialización de la medición oculta las distintas posibilidades de acceso e intensidad de uso de los medios sociales así como la visibilidad superior que tiene el Norte Global. Antes que ampliar el horizonte de posibilidades sobre la base material, se trata de nuevas herramientas que reducen las posibilidades reales de acceso a discursos distintos al del Norte Global porque amplían el espacio de igualdad sin ocuparse de las diferencias materiales sobre las que se asientan. Pero el acceso abierto o las métricas alternativas no son las únicas respuestas. Existen otras que plantean resistencias, rechazo por parte de la comunidad científica y son perseguidas por las grandes editoriales. Por el momento continúan resistiendo las reaxiomatizaciones y plantean interrogantes a los principios hegemónicos subyacentes.

\subsection{Las "apocalípticas": resistencias, ampliación del espacio e ironías}

SCI-HUB ${ }^{14}$ fue creada en 2011 por Alexandra Elbakyan, una estudiante en neurociencia kazaja frustrada por no poder acceder a los artículos de pago para realizar su investigación —evidentemente Kazajistán no se puede permitir las suscripciones para hacer ciencia buena - En 2017 contaba con el 83\% de todos los artículos de pago de la historia y es utilizada literalmente por todo el mundo (Bohannon, 2016). Al hacer disponible libremente toda la producción científica de la historia esta plataforma está cortocircuitando el modelo de suscripción de las editoriales comerciales porque atenta contra su monopolio en la prestación de servicios. Por ello en junio de 2015 RELX demandó a su creadora — de 26 años en ese momento- y en junio de 2017 fue sentenciada a una multa de 15 millones de dólares por infringir las leyes de propiedad intelectual (Schiermeier, 2017). En consecuencia, la arquitectura legal de la propiedad intelectual conduce a que la mayor biblioteca gratuita del conocimiento científico público que se ha conocido sea ilegal y su creadora condenada. Pero SCI-HUB también ha sido atacada por la comunidad del acceso abierto. Si bien Green (2017) sostiene que el éxito de SCI-HUB representa la impotencia y derrota del acceso abierto después de 20 años de lucha, otra parte de la comunidad afirma que puede ser perjudicial porque puede poner en peligro los "avances conseguidos" (Priego, 2016). Una crítica que no recogen es la posibilidad de que SCI-HUB muera de éxito y, eliminando de facto el modelo de suscripcio-

14 Ver URL: <https://sci-hub.cc/>. (Consultado el 13-10-17). 
nes, impulse aún más el modelo de PPP, reduciendo las posibilidades de publicar fuera del Norte Global.

Una segunda forma de resistencia es un conjunto relativamente difuso de estrategias editoriales que intentan mantener la calidad editorial sin estar obsesionadas por los índices. Cuando una revista es comprada por una gran editorial o sus PPP resultan exorbitantes, un número reducido pero casi constante de consejos editoriales renuncian y crean nuevas revistas con criterios más o menos abiertos. Probablemente el mayor ejemplo lo constituya el proyecto PLoS (Public Library of Science), pero también en 2016 la revista Lingua, propiedad de RELX, y la Journal of Algebraic Combinatorics, propiedad de Springer, cuyos consejos editoriales renunciaron masivamente por los PPP (1.800 y 3.000 dólares respectivamente) para refundar las revistas con criterios abiertos y sin el beneficio como criterio principal (Reller, 2015; Onwuemezi, 2015; McKenzie, 2017). Finalmente hay que señalar que la mayoría de revistas creadas por universidades a partir de 2000 son las denominadas "periféricas" porque no se encuentran recogidas dentro de los principales índices. Y que el $80 \%$ de los artículos en Iberoamérica son publicados en este tipo de revistas (Mercado Percia, 2015). Esto produce que aquellas revistas que consiguen estar indexadas reciban una cantidad de artículos que sus estructuras organizativas difícilmente pueden asumir, cuestión que las pone en la tesitura de profesionalizarse, es decir: conseguir financiación pública, cobrar PPP o formar parte de una editorial comercial que facilita la gestión.

Como colofón y a pesar de suponer menos del $2 \%$ del total de artículos publicados (Bell, 2017), las revistas predadoras (predator journals) son continuamente defenestradas y producen el escándalo de la comunidad científica en general. Aprovechando los bajos costes de edición online y el modelo de PPP, estas revistas obtienen grandes beneficios porque relajan — cuando no falsifican - los procesos de revisión por pares. De esta forma publican, previo pago, prácticamente cualquier artículo que se les envíe. Junto a estas revistas también surgen un conjunto de indicadores engañosos que refuerzan la impresión de legitimidad ${ }^{15}$. En resumen, las revistas predadoras y los índices engañosos replican la estructura del modelo de revistas con el fin de obtener beneficios económicos. Ambos cubren la necesidad - desesperación - de muchos investigadores jóvenes - el vector cognitivo- y/o del Sur Global — vector espacial - por publicar en revistas con algún tipo de impacto para promocionar sus carreras académicas (Xia et al., 2015). Esta opción no sólo es más sencilla sino también más barata.

Si bien la existencia de revistas predadoras es incuestionable el asunto es más complejo. En tanto que éstas revistas minan el modelo de los PPP podría pensarse que las big-five serían las primeras interesadas en denunciar esta situación. Pero lo cierto es que entre 2000 y 2005 RELX publicó al menos 5 revistas predadoras (Grant, 2009) e incluso los mejores economistas del mundo publicaron un 5\% de sus trabajos en revistas predadoras... sólo en 2015 (Chawla, 2016). En definitiva, conocer si una revista es o no predadora no es tarea sencilla, pero de su existencia

15 Ver URL: <http://beallslist.weebly.com/>. (Consultado el 23-11-17). 
se pueden extraer al menos dos cuestiones. En primer lugar, siendo evidente que las revistas predadoras y los índices engañosos son un claro ejemplo de mala práctica científica, es irónico que sean difíciles de detectar siguiendo los criterios objetivos que exigen los índices de impacto y la lista más exhaustiva resultara fiable porque se elaboraba a mano, no de forma automática, siguiendo criterios propios del Norte Global ${ }^{16}$. En segundo lugar, Bell (2017) y Nwagwu ( 2016) han destacado cómo éstos criterios están fuertemente basados en las posibilidades, dinámicas de trabajo y cosmovisión del Norte Global. Esto conduce a que proyectos auténticos en África o Asia sean considerados predadores por cuestiones como la capacidad técnica y el aspecto gráfico de la plataforma, la inexistencia de mujeres o la poca diversidad geográfica de su comité editorial, o que sea un única editorial la que publique la revista. Quizás sea más sencilla la diversidad geográfica, la igualdad de género y editoriales con más de una revista en países del Norte Global que en India, Pakistán, Nigeria o Turquía sin ir más lejos. Esto resulta evidente cuando se tiene en cuenta que la mayoría de las revistas calificadas como predadoras son editadas en la India, seguida de Asia, luego África y con una participación de Estados Unidos bastante destacable (Shen \& Björk, 2015; Xia et al., 2015). Este ecosistema predador compuesto por revistas e índices no puede dejar de ser visto como una parodia (Bell, 2017) que, replicando el modelo de las grandes editoriales comerciales y los índices de impacto, cubre los inmensos huecos que éstos dejan.

\section{Contra las alternativas}

En definitiva, el criterio que distingue las alternativas "integradas" de las resistencias "apocalíticas" es la capacidad que tienen, en relación a las revistas científicas y el factor de impacto, de producir espacios que se integren en las dinámicas coloniales y constrictivas actuales, o que a través de sus resistencias amplíen estos espacios. En efecto, las "alternativas" no amplían el horizonte de posibilidades ni resultan inclusivas, y su complejidad reside justamente en fingir dar una solución mejor a un problema que continúan sin resolver. Desde un punto de vista epistemológico Santos plantea la cuestión en función de los interrogantes que se proponen resolver y los efectos que producen:

Las preguntas fuertes son las que se dirigen [...] a los fundamentos que crean el horizonte de posibilidades entre las cuales es posible elegir. Por ello, son preguntas que generan una perplejidad especial. [Mientras que] las respuestas débiles son las que no consiguen reducir esa complejidad sino que, por el contrario, la pueden aumentar (2010a:7).

Siguiendo la distinción planteada sólo las resistencias "apocalípticas" producen perplejidad dentro de la comunidad académica y apuntan líneas concretas de acción

16 El propio Beall sostuvo que los criterios no podían aplicarse de forma exhaustiva ni automática. La lista de estos criterios puede encontrarse en URL: <http://beallslist.weebly.com/uploads/3/0/9/5/30958339/criteria2015.pdf>. (Consultado el 23-11-17). 
que permitan ampliar el horizonte de posibilidades (Santos, 2016). Como se verá a continuación el acceso abierto en un caso paradigmático a este respecto. Pero antes de entrar en el desarrollo del ejemplo es necesario explicar en virtud de qué ideas se operacionalizan estas dinámicas que plantea Santos. Dentro de este marco la producción de respuestas débiles, o la debilitación de respuestas fuertes, puede describirse recurriendo a Morozov y Floridi. Según el primero, una de las características principales de Internet, y por tanto de las sociedades actuales del Norte Global al menos, es el solucionismo (Morozov, 2015). Éste se refiere a la voluntad superficial de mejorar lo existente mediante la reformulación de

todas las situaciones sociales complejas como problemas con definición clara y soluciones definitivas y computables, o como procesos transparentes y obvios a primera vista que pueden optimizarse sin mayor esfuerzo - apenas contando con los algoritmos correctos [...] [L]o polémico, entonces, no es la solución planteada, sino la definición misma del problema [...] el solucionismo da por sentado el problema que intenta resolver, en lugar de investigarlo, y así llega a "a la respuesta antes de haber terminado de formular las preguntas en toda su amplitud" (2015: 24).

Desde la filosofía de la información, para Floridi (2011) el conocimiento, en tanto que intercambio de información, no consiste en obtener mensajes del mundo sino en encontrar la forma adecuada de comunicarlos con él, de las preguntas que se formulen. Estas formas serán tanto mejores, darán más información y serán más adecuadas en la medida que sean más precisas y tengan en cuenta su contexto de formulación (Floridi, 2014), porque en definitiva es lo que les da un sentido práctico. Aplicado a la ciencia, internet y el Norte Global, la forma de relacionarnos con la realidad es a través de filtros (proxys, indicadores, índices) (Floridi, 2015a), que modulan el contenido que indexan. La masificación y ubicuidad de su uso hace que este poder "gris" (Floridi, 2014; 2015b), en la sombra, cobre autonomía y termine ocultando tanto su proceso de creación como el objeto del que es vicario. Cuando se busca en Google implícitamente aceptamos su algoritmo como un filtro adecuado; cuando sólo usamos el buscador de Google para todo, se convierte en el filtro que modula las preguntas que podemos hacernos y ofrece las respuestas que nos permiten comprender la realidad; cuando se utiliza Facebook como origen para la obtención de información se asume que su algoritmo sabe qué nos interesa; cuando se utilizan las métricas para evaluar la calidad científica se pierde el carácter vicario de toda métrica y la calidad queda definida por el impacto.

Ambas filosofías, profundamente científicas, pueden aportar un marco práctico de aplicabilidad a la gestión de respuestas fuertes y débiles por parte de las sociedades del Norte Global. La ciencia avanza en la medida que descubre y predice, respondiendo. Si estas respuestas, por su progresiva aplicabilidad técnica, eliminan sus contextos de producción y uniformizan los criterios de aplicabilidad, producen nuevas preguntas necesariamente más débiles, más específicas, especializadas y progresivamente impotentes para dar respuestas eficaces a los interrogantes cada vez más fuertes planteados por las sociedades. Por supuesto que el conocimiento "está ahí" y cada vez hay y habrá más respuestas, pero cada vez más débiles. Por ello la distancia abismal de las preguntas fuertes es cada vez mayor respecto a las 
respuestas... porque están "ahí”, cada vez más cerca porque con cada vez más específicas, técnicas y abundantes, y cada vez más lejos por su incapacidad de poder dar una respuesta a los interrogantes que no dejan de generar perplejidad.

Esta dinámica fue contenida hasta cierto punto mientras el Estado, lo público, continuaba siendo el agente principal dentro de las economías y se pensaba que el planteamiento de alternativas tenía algún sentido y encontraría espacio. A medida que lo público pierde capacidad de influencia por la progresiva incorporación de agentes externos que se validan siguiendo otros criterios, el planteamiento de alternativas se modifica. Es decir, a medida que el Estado externaliza servicios, enajena su capacidad de influencia en servicios que persiguen la rentabilidad según la validación que utilicen, sea el dinero o el factor de impacto. Por ello en 1968 Iván Illich $^{17}$ mandaba "al diablo a las buenas intenciones" y proponía alternativas. Más de 40 años después, en un contexto en el que las reglas son construidas por todos los jugadores y no sólo por el Estado (Lyotard, 1990), y en el que el proyecto de la globalización obliga a todos los jugadores a asumir las mismas identidades, es decir la misma forma de validarnos en el orden social (Castro-Gómez, 2004), proponer alternativas tiene el efecto contraproducente de alimentar la máquina unificadora, marginadora e invisibilizadora de realidades (Deleuze \& Guattari, 1985).

Esta es la evolución que ha atravesado el acceso abierto. Acceder a los artículos de forma irrestricta era sólo una cuestión; por supuesto se asumía que nadie pagaría por publicar $^{18}$ y que el acceso abierto conllevaría una mayor apertura general del sistema de revistas y evaluación de la ciencia, una mayor descentralización de los sistemas de evaluación y la posibilidad de su acceso acceder a ellos. En resumen, "detrás" del acceso abierto estaba el uso de software libre para publicar revistas online y que todas las personas tuvieran acceso a las mismas, y con una arquitectura que posibilitara el compartir información para elaborar los indicadores y métricas que se estimara. No se trataba de una alternativa sino de otro modelo de entender y hacer ciencia. La actualidad es muy distinta y el acceso abierto está cada vez más lejos de generar comunidades de conocimiento que cooperen y aporten sus resultados al conocimiento común. Ha sido impulsado en la medida que conseguía ser integrado dentro de las estructuras de validación predominantes, dejando el cascarón y vaciando el contenido. Se trata de una dinámica gatopardista en la que se alteran los términos pero se mantiene el modelo: la comunidad tiene lo que históricamente demandaba, el acceso abierto a cada vez más publicaciones, pero transformado en que el acceso a publicar es cada vez más restrictivo. Que la industria de las editoriales comerciales sea uno de los negocios más rentables y que menos valor añade al producto final puede indignar, pero dentro de sociedades capitalistas culpabilizar a una corporación por cumplir sus fines no tiene sentido. Los desorbitados precios de los PPP encuentran su justificación en la hegemonía del factor de impacto como validador, filtro y creador de realidad científica. Por el contrario, SCI-HUB y las revistas predadoras, utilizando las mismas estrategias amplían y reaxiomatizan el horizonte de posibilidades. No alteran ningún proceso

17 Ver URL: <https://www.ivanillich.org.mx/buenas.pdf>. (Consultada el 11-02-18).

18 Recuérdese la furiosa negativa de Wittgenstein acerca de costear la publicación de su propio Tractatus en la editorial de von Ficker Der Brenner, una forma curiosa de PPP antigua. Finalmente se publicó gratuitamente en inglés en Routledge, Kegan \& Paul. Es decir, seguramente el libro filosófico más importante del siglo XX, junto a Ser y Tiempo, supuso cero beneficio para su autor (Monk, 1997). 
ni ofrecen servicios u opciones alternativas. Ofrecen el mismo servicio y, contraponiéndose a las vías gerencialmente aceptadas, activan toda la operativa legal, institucional y coercitiva de persecución y condena.

De manera similar, el uso de la teoría normativa — que no real- mertoniana y de la Ley de Bradford invisibiliza las condiciones materiales y éticas del Norte Global porque al presentarlas como universales las naturaliza (Castro-Gómez, 2010). Y al ser asumido como el validador de la ciencia a nivel mundial, se institucionalizan una serie de valores y naturalizan que institucionalizan la marginación del Sur Global. El mismo Garfield, conocedor de la performatividad de su índice, en 1997 reclamó la necesidad de una "definición estadísticamente válida de sesgo" (1997) para responder a las críticas que, ya entonces, se hacían sobre la exclusión e infrarrepresentación de revistas de países del Sur Global. Ni Google Scholar ni las altmetrics prestan el mismo servicio de otra forma, sino que complementan y amplían los criterios de evaluación. A la "igualdad" de la Ley de Bradford superponen y añaden la "igualdad" de una miríada de medios sociales y documentos. Se analiza más pero no mejor. De esta forma la pregunta fuerte sobre cómo medir la ciencia tiene cada vez más respuestas que, en lugar de contribuir a la convivencialidad y reducir la complejidad, la aumentan aún más, En definitiva se trata de nuevos instrumentos estadísticos que generan supuestamente nuevas realidades sociales para continuar produciendo la misma desigualdad social, pero aumentada.

\section{Conclusiones. Eppur continua a muoversi}

Como se ha visto, los principios de metrificación de la realidad y gestión de la vida han sido parte de la evolución del Norte Global desde los inicios de la ciencia. Y la estructura actual encuentra su inicio en el final de la Segunda Guerra Mundial. A partir de este momento comenzará el despliegue del fascismo epistemológico (Santos, 2010a, 2016): toda una serie de procesos objetivos a través de los cuales el conocimiento científico avanza, en términos prácticos, marginando otros saberes diferentes del científico así como disciplinas científicas como las artes y las humanidades. Dentro de esta dinámica en la que la evaluación de la producción científica será cada vez más importante, su desarrollo concreto conducirá a que las revistas científicas y los índices de impacto tengan un rol cada vez más hegemónico dentro de todo este proceso.

A través de las grandes editoriales comerciales y los servicios de impacto se crea un círculo vicioso que se alimenta a sí mismo, como una gran estafa piramidal, en una dinámica viciosa que necesariamente excluye y debilita una cantidad cada vez mayor de conocimientos. No puede afirmarse que se trate de una estrategia consciente. Más bien forma parte de un Sistema-Mundo capitalista que, para continuar funcionando, necesita fagocitar lo que existe y gerencializar, metrificar y mercantilizar cualquier fenómeno social como es la ciencia misma. De esta forma un artefacto roto, en crisis permanente y cada vez más exiguo como los sistemas públicos de ciencia, crónicamente recortados, a pesar de todo "se siguen moviendo" cada vez más rápido. El conocimiento científico avanza con ese doble movimiento por el cual discrimina cada vez más partes del conocimiento a través de su expansión por medio de temas de investigación cada vez más débiles. Y para ello es ne- 
cesario una ampliación en la oferta de servicios que continúe reduciendo las opciones disponibles: en los últimos años RELX ha ampliado su expansión y además de revistas ha comprado repositorios de contenido como SSNR y bepress, el gestor de referencias Mendeley y su servicio de impacto. SCOPUS, incluye cada vez más revistas. Clarivate, por su parte, ha adquirido SciELO y además de elaborar índices regionales crea otros más globales, como el Emerging Sources Citation Index, que persiguen continuar concentrando la medición del impacto. Las revistas no son el final, son sólo el inicio.

Por ello la intención de materializar las epistemologías, aplicándolas a las revistas científicas, en la medida que son el formato concreto de medición, tiene la intención de destacar que la generación de conocimientos alternativos que no resistan las validaciones hegemónicas actuales sólo ahondarán más la brecha entre el Norte y Sur Globales. Países como Brasil (Ferreira et al., 2013), Colombia (Chavarro et al., 2017), España (Van Leeuwen \& Wouters, 2017), China (Quan et al., 2017), India o Malasia (Abrizah \& Thelwall, 2014), por nombrar sólo algunos, toman los índices de impacto top progresivamente como los únicos aceptables para su personal universitario. No tiene sentido crear Universidades o Centros de Conocimientos con epistemologías del Sur si los conocimientos que produzcan tendrán que ser validados dentro de un sistema en el que sistemáticamente perderán posiciones y capacidad para influir en las reglas del juego. En la actualidad, la presión por publicar en países del Sur Global es cada vez más fuerte dentro de un contexto, como se ha visto, progresivamente desfavorable. Por ello las buenas voluntades, las alternativas y las propuestas sólo sirven para que éstas sean integradas dentro del Sistema Mundo que se expande discriminando cada vez más partes de la realidad. Por esta razón aquí no se elabora ninguna propuesta: no se trata de una cuestión técnica, las tecnologías para la resistencia y la creación de otras realidades están disponibles; se trata de trabajar con prácticas que materialicen las resistencias y los marcos epistemológicos que impidan que éstas se transformen en alternativas.

\section{Bibliografía}

Abrizah, A. \& Thelwall, Mike (2014) "Can the impact of non-Western academic books be measured? An investigation of Google Books and Google Scholar for Malaysia". Journal of the Association for Information Science and Technology, vol. 65, núm. 12, 24982508.

Bell, Kirsten (2017) “«Predatory» Open Access Journals as Parody: Exposing the Limitations of «Legitimate» Academic Publishing". tripleC: Communication, Capitalism \& Critique. Open Access Journal for a Global Sustainable Information Society, vol. 15, núm. 2, 651-662.

Beverungen, Armin; Böhm, Steffen \& Land, Christopher (2012) "The poverty of journal publishing". Organization, vol. 19, núm. 6, 929-938.

Björk, Bo-Christer \& Solomon, David (2015) "Article processing charges in OA journals: relationship between price and quality". Scientometrics, vol. 103, núm. 2, 373-385.

Bohannon, John (2016) “Who's downloading pirated papers? Everyone”. Science, vol. 352, núm. 6285, 508-512.

Bradford, Samuel C. (1976) "Sources of Information on Specific Subjects". Collection Management, vol. 1, núm. 3-4, 95-104. 
Buranyi, Stephen (2017) "Is the staggeringly profitable business of scientific publishing bad for science?" [Puesto en línea el 29 de septiembre de 2010. URL: $<$ http://www.theguardian.com/science/2017/jun/27/profitable-business-scientificpublishing-bad-for-science $>$. Consultado el 31 de octubre de 2017].

Burnham, James (1945) The managerial revolution or what is happening in the world now. Westport (Connecticut): Greenwood.

Bush, Vannevar (1945a) "As we may think". The Atlantic Monthly, vol. 176, núm. 1, 101108.

Bush, Vannevar (1945b) Science, the endless frontier: A report to the President. U. S. Govt. print. [URL: <https://www.nsf.gov/about/history/nsf50/vbush1945.jsp>. Consultado el 31 de octubre de 2017].

Castro-Gómez, Santiago (2004) "El problema de la «invención del otro»", en S. Dube, I. Banerjee Dube y W. D. Mignolo (coords.) Modernidades coloniales. Otros pasados, historias presentes. México D. F.: El Colegio de México / Centro de Estudios de Asia y África, 88-98.

Castro-Gómez, Santiago (2010) La hybris del punto cero: ciencia, raza e ilustración en la Nueva Granada (1750-1816). Bogotá: Editorial Pontificia Universidad Javeriana.

Chavarro, Diego; Tang, Puay \& Ràfols, Ismael (2017) "Why researchers publish in nonmainstream journals: Training, knowledge bridging, and gap filling". Research Policy, vol. 46, núm. 9, 1666-1680.

Chawla, Dalmeet Singh (2016) "Even top economists publish in predatory journals, study finds". Retraction Watch. [Puesto en línea el 27 de octubre de 2016. URL: $<$ http://retractionwatch.com/2016/10/27/even-top-economists-publish-in-predatoryjournals-study-finds/>. Consultado el 31 de octubre de 2017].

Cole, Stephen (2004) “Merton's Contribution to the Sociology of Science”. Social Studies of Science, vol. 34, núm. 6, 829-844.

Cookson, Robert (2015) "Elsevier leads the business the internet could not kill". The Financial Times. [Puesto en línea el 15 de noviembre de 2015. URL: $<$ https://www.ft.com/content/93138f3e-87d6-11e5-90de-f44762bf9896>. Consultado el 31 de octubre de 2017].

Crane, Diana (1967) "The gatekeepers of science: Some factors affecting the selection of articles for scientific journals". The American Sociologist, vol. 2, núm. 4, 195-201.

Declaración de Budapest (1999) Declaración sobre la Ciencia y el uso del saber científico. [URL: <http://www.oei.es/historico/salactsi/budapestdec.htm>. Consultado el 31 de octubre de 2017].

Deleuze, Gilles \& Guattari, Félix (1985) El antiedipo: capitalismo y esquizofrenia. Barcelona: Paidós

Deutsche Bank (2005) Reed Elsevier moving the supertanker. Berlín: Company focus (Global Equity Research Report 36).

Echeverría, Javier (2014) Innovation and values: a European perspective. Reno: Center for Basque Studies.

Eco, Umberto (2015) Apocalipticos e integrados. Barcelona: Debolsillo.

Reller, Tom (2015) "Addressing the resignation of the Lingua editorial board". Elsevier Connect. [Puesto en línea el 4 de noviembre de 2015. URL: $<$ https://www.elsevier.com/connect/addressing-the-resignation-of-the-lingua-editorialboard $>$. Consultado el 31 de octubre de 2017].

Fanelli, Daniele (2009) "How Many Scientists Fabricate and Falsify Research? A Systematic Review and Meta-Analysis of Survey Data". PLoS ONE, vol. 4, num. 5, p. e5738. 
Fanelli, Daniele (2014) "Publishing: Rise in retractions is a signal of integrity". Nature, vol. 509, num. 7498, 33.

Fanelli, Daniele; Costas, Rodrigo \& Larivière, Vincent (2015). "Misconduct Policies, Academic Culture and Career Stage, Not Gender or Pressures to Publish, Affect Scientific Integrity". PLoS ONE, vol. 10, num. 6, p. e0127556.

Ferreira, Renata C.; Antoneli, Fernando \& Briones, Marcelo R. S. (2013) "The hidden factors in impact factors: a perspective from Brazilian science". Frontiers in Genetics, vol. 4, artículo 130.

Floridi, Luciano (2011) "A defence of constructionism: Philosophy as conceptual engineering”. Metaphilosophy, vol. 42, núm. 3, 282-304.

Floridi, Luciano (2014) The fourth revolution: How the infosphere is reshaping human reality. Oxford: Oxford University Press.

Floridi, Luciano (2015a) “A Proxy Culture”. Philosophy \& Technology, vol. 28, núm. 4, 487-490.

Floridi, Luciano (2015b) “The New Grey Power”. Philosophy \& Technology, vol. 28, núm. 3, 329-332.

Foucault, Michel (2014). Seguridad, territorio, población: curso en el Collége de France (1977-1978). México D. F.: Fondo de Cultura Económica.

Garfield, Eugene (1955) "Citation Indexes for Science: A New Dimension in Documentation through Association of Ideas". Science, vol. 122, núm. 3159, 108-111.

Garfield, Eugene (1979) "Scientometrics comes of age". Current Contents, núm. 46, 5-10.

Garfield, Eugene (1997) "A statistically valid definition of bias is needed to determine whether the Science Citation Index discriminates against third world journals". Current Science, vol. 73, núm. 8, 639-641.

Grant, Bob (2009) "Elsevier published 6 fake journals". The Scientist. [Puesto en línea el 7 de mayo de 2009. URL: <http:/www.thescientist.com/?articles.view/articleNo/27383/title/Elsevier-published-6-fake-journals/>. Consultado el 31 de octubre de 2017].

Gray, Chris Hables (2002) Cyborg Citizen: Politics in the Posthuman Age. New York: Routledge.

Green, Toby (2017) “We've failed: Pirate black open access is trumping green and gold and we must change our approach". Learned Publishing, vol. 30, núm. 4, 325-329.

Gross, Alan G.; Harmon, Joseph E. \& Reidy, Michael S. (2002) Communicating science: the scientific article from the 17th century to the present, Oxford: Oxford University Press.

Harzing, Anne-Wil \& Alakangas, Satu (2016) "Google Scholar, Scopus and the Web of Science: a longitudinal and cross-disciplinary comparison”. Scientometrics, vol. 106, núm. 2, 787-804.

Hayes, John (1995) “The Internet's first victim? (Academic publisher Reed Elsevier; electronic publishing infringes on traditional publishing services) (Industry Trend or Event) | HighBeam Business: Arrive Prepared”. Forbes, 18 de Diciembre de 1995, 200-201.

Klein, Naomi (2010) La Doctrina del Shock. Madrid: Grupo Planeta.

Larivière, Vincent; Archambault, Éric; Gingras, Yves \& Vignola-Gagné, Étienne (2006) "The place of serials in referencing practices: Comparing natural sciences and engineering with social sciences and humanities". Journal of the American Society for Information Science and Technology, vol. 57, núm. 8, 997-1004.

Larivière, Vincent; Haustein, Stefanie \& Mongeon, Philippe (2015) "The Oligopoly of Academic Publishers in the Digital Era”. PLOS ONE, vol. 10, núm. 6, p. e0127502. 
Lyotard, Jean-François (1990) La condición postmoderna: informe sobre el saber. México D. F.: Red Editorial Iberoamerica (REI).

MacRoberts, Michael (1997) "Rejoinder". Journal of the American Society for Information Science, vol. 48, núm. 10, p. 963-963a.

MacRoberts, Michael \& MacRoberts, Barbara (1996) "Problems of citation analysis". Scientometrics, vol. 36, núm. 3, 435-444.

McKenzie, Lindsay (2017) "Math journal editors resign to start rival open-access journal". Inside HigherEd. [Puesto en línea 31 de julio de 2017. URL: $<$ https://www.insidehighered.com/news/2017/07/31/math-journal-editors-resign-startrival-open-access-journal $>$. Consultado el 31 de octubre de 2017].

Mercado Percia, Heiner (2015) "Métricas fraudulentas y las revistas iberoamericanas. Entrevista a Rafael Repiso". Journals \& Authors [Puesto en línea 18 de octubre de 2015. URL: <http://jasolutions.com.co/calidad-editorial/metricas-fraudulentas-y-las-revistasiberoamericanas-entrevista-a-rafael-repiso/>. Consultado el 31 de octubre de 2017].

Merton, Robert King \& Storer, Norman W. (1985) La sociología de la ciencia: investigaciones teóricas y empíricas. Madrid: Alianza Editorial.

Mingers, John \& Leydesdorff, Loet (2015) "A review of theory and practice in scientometrics". European Journal of Operational Research, vol. 246, núm. 1, 1-19.

Mignolo, Walter (2005) "Cambiando las éticas y las políticas del conocimiento: lógica de la colonialidad y postcolonialidad imperial”. Tabula Rasa, núm. 3, 47-72.

Mignolo, Walter (2013) "Geopolítica de la sensibilidad y del conocimiento. Sobre (de)colonialidad, pensamiento fronterizo y desobediencia epistémica". Revista de filosofía, vol. 74, núm. 2, 7-23.

Monk, Ray (1997) Ludwig Wittgenstein: el deber de un genio. Barcelona: Anagrama.

Morozov, Evgeny (2015) La locura del solucionismo tecnológico. Buenos Aires: Katz.

Nwagwu, W. E. (2016) "Open Access in the Developing Regions: Situating the Altercations About Predatory Publishing / L'accès libre dans les régions en voie de développement: Situation de la controverse concernant les pratiques d'édition déloyales". Canadian Journal of Information and Library Science, vol. 40, núm. 1, 58-80.

Onwuemezi, Natasha (2015) "Editorial staff quit Elsevier journal en masse". The Bookseller. [Puesto en línea el 5 de noviembre de 2015. URL: $<$ https://www.thebookseller.com/news/entire-editorial-staff-elseiver-journal-quit316002>. Consultado el 31 de octubre de 2017].

Orwell, George (1955) James Burnham and the managerial revolution. Berkeley (California): Berkeley Young Socialist League.

Pinfield, Stephen; Salter, Jennifer \& Bath, Peter A. (2016) “The «total cost of publication» in a hybrid open-access environment: Institutional approaches to funding journal articleprocessing charges in combination with subscriptions". Journal of the Association for Information Science and Technology, vol. 67, núm. 7, 1751-1766.

Price, Derek J. de Solla (1951) "Quantitative measures of the development of science". Archives intenationales d' Historie des Sciences, vol. 4, núm. 14, 85-93.

Price, Derek J. de Solla (1961) "Science since babylon". American Journal of Physics, vol. 29, núm. 12, 863-864.

Price, Derek J. de Solla (1978) "Editorial statements”. Scientometrics, vol. 1, num. 1, 3-8.

Price, Derek J. de Solla (1986) Little Science, Big Science and Beyond. New York: Columbia University Press.

Priego, Ernesto (2016) "Signal, not solution: Notes on why Sci-Hub is not opening access". The Winnower, vol. 3, p. e145624. 
Quan, Wei; Chen, Bikun \& Shu, Fei (2017) "Publish or impoverish: An investigation of the monetary reward system of science in China (1999-2016)". Aslib Journal of Information Management, vol. 69, núm. 5, 1-18. [URL: <https://arxiv.org/abs/1707.01162>. Consultado el 31 de octubre de 2017].

Repiso, Rafael \& Torres-Salinas, Daniel (2016) "Características e implicaciones del índice de citas". EC3metrics. Evaluación científica. [Puesto en línea el 2 de febrero de 2016. URL: <https://ec3metrics.com/thinkepi-caracteristicas-e-implicaciones-del-indice-decitas-emerging-source-citation-index-thomson-reuters-las-revistas-en-estadotransitorio/>. Consultado el 31 de octubre de 2017].

Santos, Boaventura de Sousa (2010a) Para descolonizar Occidente: más allá del pensamiento abismal. Buenos Aires: CLACSO: Prometeo Libros.

Santos, Boaventura de Sousa (2010b) Descolonizar el saber, reinventar el poder. Montevideo: Ediciones Trilce

Santos, Boaventura de Sousa (2016) "The University at a Crossroads", en R. Grosfoguel, R. Hernández y E. Rosen Velásquez (eds.) Decolonizing the Westernized University. Interventions in Philosophy of Education from Within and Without. Lanham (Maryland): Lexington Books, 3-14.

Santos, Boaventura de Sousa \& Meneses, María Paula (eds.) (2014) Epistemologías del Sur. Madrid: Akal.

Schiermeier, Quirin (2017) "US court grants Elsevier millions in damages from Sci-Hub". [Puesto en línea el 22 de junio de 2017. URL: $<$ https://doi.org/10.1038/nature.2017.22196>. Consultado el 31 de octubre de 2017].

Shen, Cenyu \& Björk, Bo-Christer (2015) “«Predatory» open access: a longitudinal study of article volumes and market characteristics". BMC Medicine, vol. 13, p. 230.

Sokal, Alan D (1996) "Transgressing the boundaries: Toward a transformative hermeneutics of quantum gravity". Social Text, núm. 46/47, 217-252.

Sokal, Alan D; Bricmont, Jean \& Dawkins, Richard (1998) Intellectual impostures. Londres: Profile London.

Solomon, David J. \& Björk, Bo-Christer (2012) "Publication fees in open access publishing: Sources of funding and factors influencing choice of journal". Journal of the association for information science and technology, vol. 63, núm. 1, 98-107.

Solomon, David J. \& Björk, Bo-Christer (2016) "Article processing charges for open access publication - the situation for research intensive universities in the USA and Canada". PeerJ, vol. 4, p. e2264.

Sugimoto, Cassidy (2015). "«Attention is not impact» and other challenges for altmetrics". Wiley Exchanges. [Puesto en línea el 23 de junio de 2015. URL: $<$ https:/hub.wiley.com/community/exchanges/discover/blog/2015/06/23/attention-isnot-impact-and-other-challenges-for-altmetrics $>$. Consultado el 31 de octubre de 2017].

The PLoS Medicine Editors (2006) "The Impact Factor Game”. PLoS Med, vol. 3, núm. 6, p. e291.

Van Leeuwen, Thed N. \& Wouters, Paul F. (2017) “Analysis of Publications on Journal Impact Factor Over Time". Frontiers in Research Metrics and Analytics, vol. 2, artículo 4.

Waltman, Ludo (2015). "A review of the literature on citation impact indicators". arXiv:1507.02099 [cs.DL]. [URL: <https://arxiv.org/abs/1507.02099>. Consultado el 12 de enero de 2018].

Wouters, P. \& Leydesdorff, L. (1994) “Has Price's dream come true: Is scientometrics a hard science?” Scientometrics, vol. 31, núm. 2, 193-222. 
Xia, Jingfeng; Harmon, Jennifer L.; Connolly, Kevin G.; Donnelly, Ryan M.; Anderson, Mary R. \& Howard, Heather A. (2015) "Who publishes in «predatory» journals?". Journal of the Association for Information Science and Technology, vol. 66, núm. 7, 1406-1417.

Zuckerman, Harriet \& Merton, Robert K. (1971) "Patterns of evaluation in science: Institutionalisation, structure and functions of the referee system". Minerva, vol. 9, núm. 1, 66-100. 